\title{
Pacientes com insuficiência renal crônica em hemodiálise: tratamento e diagnóstico
}

\author{
Patients with chronic kidney insufiência in hemodialysis: pharmacotherapy and \\ diagnosis
}

\author{
Francy Bruna Nascimento de Sousa ${ }^{1}$, Wellison Amorim Pereira ${ }^{2}$, Elizângela Araújo Pestana \\ Motta ${ }^{3}$.
}

Resumo: Introdução. Os rins são órgãos complexos, de grande importância e muitas funções, controla o volume dos líquidos além de produzir e secretar hormônios: a eritropoetina, a vitamina $D$ e a renina. A doença renal crônica (DRC) é um problema de saúde pública e médica, no Brasil, a incidência e a prevalência de falência de função renal são crescentes com prognóstico ruim e os custos do tratamento altíssimos. Objetivo. Apresentar os principais marcadores bioquímicos, os medicamentos utilizados e os tipos de tratamentos realizados na insuficiência renal crônica. Metodologia. Foi realizada revisão de literatura sistemática em artigos, teses de doutorado, dissertação de mestrado e casos controles com abordagem no diagnóstico, farmacoterapêutica e principais tratamentos da insuficiência renal crônica nos bancos de dados Biblioteca Virtual de Saúde, Bireme e LILACs. Foram encontrados 125 artigos e utilizados apenas 45; os critérios de inclusão adotados foram: artigos que abordassem apenas sobre a insuficiência renal crônica, doenças de base, métodos de diagnósticos e tipos de tratamentos paliativos medicamentosos, não medicamentoso e definitivo; já os critérios de exclusão foram: trabalhos que abordassem sobre insuficiência renal aguda, doença renal em crianças, doenças que não fossem as de base e demoais públicos. grávidas com enfoque nos objetivos citados, apresentando como maior indicação para os exames bioquímicos a taxa de filtração glomerular (TFG),dosagem sérica de ureia e creatinina e a cistatina $C$, tratamento de hemodiálise (HD) e farmacoterapia com eritropoetina, andrógeno, estatinas e vitamina B12. Resultados e Conclusão. Portanto a pesquisa pôde mostrar que para se concluir um diagnóstico de insuficiência renal crônica existem os principais marcadores bioquímicos, bem como tratamentos medicamentoso e não medicamentoso para melhor terapia do paciente.

Palavras-chave: Hemodiálise; Diálise; Insuficiência Renal Crônica; Farmacoterapia; Diagnóstico.

\begin{abstract}
Introduction. The kidneys are complex organs, of great importance and many functions, controls the volume of the liquids besides producing and secreting hormones: erythropoietin, vitamin $D$ and renin. Chronic kidney disease (CKD) is a public and medical health problem in Brazil, the incidence and prevalence of renal function failure are increasing with poor prognosis and very high treatment costs. Objective. To present the main biochemical markers, the medications used and the types of treatments performed in chronic renal failure. Methodology. A systematic literature review was carried out in articles, doctoral theses, master dissertation and control cases with a diagnostic approach, pharmacotherapeutic and main treatments of chronic renal failure in the databases of the Virtual Health Library, Bireme and LILACs. We found 125 articles and used only 45; inclusion criteria were: articles dealing only with chronic renal insufficiency, basic diseases, diagnostic methods and types of medicated, non-medicated and definitive palliative treatments; the exclusion criteria were: studies dealing with acute renal failure, renal disease in children, non-basic diseases and public diseases. (GFR), urea and creatinine serum levels and cystatin C, hemodialysis (HD) treatment and pharmacotherapy with erythropoietin, androgens, statins and b12 vitamin. Results and Conclusion. Therefore the research could show that to conclude a diagnosis of chronic renal failure there are the main biochemical markers, as well as drug and non-drug treatments for better therapy of the patient.
\end{abstract}

Keywords: Hemodialysis; Dialysis; Chronic Renal Insufficiency; Pharmacotherapy; Diagnosis.

\footnotetext{
${ }^{1}$ Farmacêutica. Instituto Florence.

2 Mestrando em Biologia Parasitária. UNICEUMA

${ }^{3}$ Doutoranda em Ciências da Saúde (UFMA). Membro do CEP da UNICEUMA.
}

Autor de correspondência: E-mail: elifarmabr@gmail.com

Endereço: Rua Rio Branco, 216 - Centro, São Luís - MA, 65020-470. Telefone: (98) 98413-8428 


\section{Introdução}

Os rins são órgãos complexos, de grande importância e muitas funções, como a eliminação de resíduos tóxicos produzidos pelo organismo humano - a ureia e o ácido úrico, filtra, limpa e faz a depuração. $O$ órgão controla o volume dos líquidos, portanto, qualquer excesso de água no corpo será eliminado pela urina, o chamado efeito diurético. O rim exerce controle sobre os sais de nosso corpo, eliminando os seus excessos ou poupando-os nas situações de carência. Ele produz e secreta hormônios: a eritropoetina, a vitamina $D$ e a renina ${ }^{1}$.

A doença renal crônica (DRC) corresponde um problema de saúde pública e médica, devido a casos crescentes registrados anualmente de pacientes em tratamento hemodialítico, assim como às elevadas taxas de mortalidade, o que vem alarmando a comunidade científica internacional nas duas últimas décadas. No Brasil, a incidência e a prevalência de falência de função renal são crescentes com prognóstico ruim e os custos do tratamento altíssimos ${ }^{2}$.

Estima-se que haja no país aproximadamente 100.400 pacientes em tratamento dialítico. Destes, perto de $62,6 \%$ têm entre 19 e 64 anos, portanto, em uma faixa etária produtiva. A taxa de mortalidade de pacientes em diálise em 2013 foi de 17,9\%. Calculase que $84 \%$ dos tratamentos dialíticos, em 2013, foram custeados pelo Sistema Único de Saúde?

Apesar do número crescente de novos pacientes em diálise, o número de unidades de diálise no Brasil não vem acompanhando esse crescimento expressivo de pacientes. No Maranhão as unidades estão trabalhando com o máximo de sua capacidade, estabelecidos pelos tetos financeiros ${ }^{3}$.

A insuficiência renal crônica é definida como uma síndrome provocada por uma variedade de nefropatias que, devido a sua evolução progressiva, determinam de modo gradativo e quase sempre inexorável uma redução global das múltiplas funções renais, isto é, glomerulares, tubulares e endócrinas ${ }^{4}$.

Essa patologia crônica causa uma grande perda do número de néfrons que são destruídos ou lesados, de maneira que os que restaram não podem desempenhar as funções normais dos rins. Em consequência, os rins tornam-se incapazes de desempenhar suas múltiplas e essenciais atividades homeostáticas, onde o aumento de doenças crônicas e incapacitações na população em geral principalmente na idosa se associa com o aumento da realização de exames, uso de medicações, hospitalizações e institucionalização, com consequente enorme sobrecarga financeira ao sistema de saúde pública em todos os países ${ }^{4}$.

O tratamento em hemodiálise (HD) é realizado com o auxílio de uma máquina chamada rim artificial e os meios de tratamentos utilizados no paciente nefropata crônico são: terapia renal substitutiva, por meio da hemodiálise (HD), diálise peritoneal ambulatorial contínua (CAPD), diálise peritoneal cíclica, diálise peritoneal intermitente e transplante. O foco da nefrologia é a terapia renal substitutiva (TRS) - diálise e transplante renal como forma estabelecida de tratamento para os pacientes que evoluíam para doença renal crônica em estágio terminal (DRET). Ressalta-se também a importância de realizar o tratamento com a dieta, restringir o consumo hídrico e o uso de medicamentos ${ }^{5}$.

Durante a fase de tratamento, os portadores de insuficiência renal crônica podem ter a qualidade de vida alterada, pois existe a ansiedade prévia e no momento do tratamento, há perda da autonomia, há dificuldade em lidar 
com uma doença irreversível e incurável ${ }^{6}$.

Em relação à terapia nutricional o objetivo é manter o adequado consumo de energia, proteína, vitaminas e minerais, controlar a ingestão de sódio, potássio e líquidos para evitar o desequilíbrio eletrolítico e o edema, ponderar a ingestão da vitamina $D$, cálcio e fósforo para evitar a osteodistrofia renal e propiciar ao paciente uma dieta adequada de acordo com seu estilo de vida ${ }^{7}$.

O termo Insuficiência Renal Crônica (IRC) é utilizado para descrever o estágio de disfunção renal, que se classifica em cinco estágios variando de leve a grave avaliado pela taxa de filtrado glomerular, calculada a partir da depuração da creatinina endógena ou depuração de creatinina, que varia de leve a grave. A taxa de filtração glomerular (TFG) é a melhor medida geral da função renal e a mais facilmente compreendida pelos médicos e pacientes. Ela é definida como a capacidade dos rins de eliminar uma substância do sangue e é expressa como o volume de sangue que é completamente depurado em uma unidade de tempo. Normalmente, o rim filtra o sangue e elimina os produtos finais do metabolismo proteico, enquanto preserva solutos específicos, proteínas (particularmente albumina) e componentes celulares ${ }^{8} 9$.

As manifestações clínicas mais importantes desta doença são: a fraqueza, adinamia (fraqueza muscular) e fadiga fácil que se correlaciona com o grau de anemia, queixas de prurido, edema e pele facilmente escoriável, anorexia, náuseas e vômitos são sintomas iniciais que se agravam com a relação dos níveis de azotemia (elevação plasmática), dispneia progressiva, dor retroesternal(dor torácica não cardiogênica) que pode estar presente devido à pericardite, nictúria, dor, dormência e câimbras nas pernas, impotência e perda da libido, irritabilidade fácil e incapacidade de concentração ${ }^{1}$.

Entende-se que a insuficiência renal crônica é uma patologia que atinge grande parte da população, e o transplante renal ajuda na recuperação do paciente para que tenha a saúde mais próxima da normalidade, porém isso tem representado o principal desafio, contudo não é fácil, em relação a isso busca-se identificar quais os tratamentos paliativos medicamentoso e não medicamentoso mais utilizados nos pacientes renais crônicos. Enfim, com o estudo em referência objetiva-se apresentar os principais marcadores bioquímicos e os tratamentos em geral (medicamentoso

medicamentoso).

\section{Metodologia}

Trata-se de um estudo de revisão sistemática, dando ênfase no diagnóstico, e nos principais tratamentos medicamentoso e não medicamentoso da insuficiência renal crônica. O período da pesquisa teve início em novembro de 2015 e o período da coleta em março a maio de 2016. Foi realizado a pesquisa em artigos, teses de doutorado dissertação de mestrado e casos controles nas bases de dados indexada através da Biblioteca Virtual da Saúde (BVS), Bireme, Scielo, LILACS. Em seguida foram estabelecidos critérios de inclusão e exclusão para refinar os resultados encontrados.

Utilizou-se como critério de inclusão artigos que abordassem sobre insuficiência renal crônica, portadores de doenças que não fossem as de base, métodos de diagnósticos e tipos de tratamentos paliativos medicamentosos, não medicamentosos e definitivo. E os 
critérios de exclusão foram: trabalhos que abordassem sobre insuficiência renal aguda, ou doença renal em crianças, ou doenças que não fossem a de base e grávidas.

A abrangência temporal dos estudos foi definida entre os anos de 2010 a 2015 e, o idioma, somente textos em português. Foram pesquisados no total de 125 artigos, teses, dissertações e casos controles, porém só foram utilizados apenas 45 que discorriam sobre o diagnóstico, famacoterapêutica e tratamento dialítico, encontrados nos títulos e nos resumos dos artigos. A busca foi feita por meio de palavras chaves: hemodiálise, diálise, doença renal crônica, farmacoterapêutica, diagnóstico.

\section{Resultados e discussão}

Avaliar a função renal é fundamental para realizar o diagnóstico e proceder ao tratamento adequado para doença renal crônica, o tratamento ideal da insuficiência renal crônica (IRC) é baseado em três pilares: diagnóstico precoce da doença; encaminhamento imediato para tratamento nefrológico e implementação de medidas para preservar a função renal ${ }^{13} 15$.

$\mathrm{Na}$ tabela 1 apresentam-se os principais exames marcadores para diagnosticar a insuficiência renal crônica baseado nas referências.

O principal marcador fisiológico da insuficiência renal crônica (IRC) é a filtração glomerular, a qual estima a perda da função renal. À medida que a insuficiência renal progride, (TFG) diminui, com a queda progressiva da (TFG) consequente perda das funções regulatórias, excretórias e endócrinas, ocorre 0 comprometimento de essencialmente todos os outros órgãos do organismo ${ }^{614}$.
Outros marcadores que por sua vez fazem parte de forma acentuada no diagnóstico da IRC é a creatinina que é produzida pelos rins, fígado e pâncreas, ela fornece dados importantes para avaliação da função renal principalmente para a filtração glomerular e um diagnóstico preciso é utilizada como marcador útil, a uréia é o soluto mais osmoticamente ativo, desequilibrando o organismo quando esta é removida rapidamente, os níveis desses resíduos aumentam quando diminui a taxa de filtração glomerular, pois a uréia é reabsorvida pelo túbulo renal após o processo de filtração, o que não acontece com a creatinina ${ }^{1516}$.

A depuração de creatinina é diretamente proporcional à geração de creatinina e inversamente proporcional à sua concentração sanguínea. $A$ vantagem da depuração da creatinina sobre a depuração das substâncias padrão ouro é o fato de ser a creatinina de produção endógena, sua determinação é de baixo custo e é realizada na maioria dos laboratórios de análises clínicas ${ }^{17}$.

A cistatina $C$ é filtrada livremente pelo glomérulo renal. Os principais atributos da cistatina $\mathrm{C}$, como marcador bioquímico para avaliação da filtração glomerular, são o pequeno tamanho e o alto ponto isoelétrico de características essas que facilitam a filtração através da membrana glomerular diferentemente da dosagem de ureia e creatinina, a concentração sérica da cistatina $C$ não sofre influencia do volume da massa muscular, do sexo ou da alimentação, é útil nos casos em que a medida da creatinina não é adequada. A cistatina C é quase completamente catabolizada no túbulo proximal, assim como outras proteínas de baixo peso molecular. A cistatina $\mathrm{C}$ e a glicoproteína (PBT) consideradas marcadores mais sensíveis da taxa de filtração glomerular (TFG) quando comparadas 
Tabela 1. Principais marcadores para diagnóstico patológico da insuficiência renal crônica.

\begin{tabular}{|c|c|c|}
\hline Tipos de exames & $\begin{array}{l}\text { Valores de referência para } \\
\text { diagnóstico da DRC }\end{array}$ & Referência \\
\hline $\begin{array}{l}\text { TGF - Taxa de Filtração } \\
\text { Glomerular } \\
\text { Dosagem Sérica de Uréia } \\
\text { e Creatinina } \\
\text { Depuração da Creatinina }\end{array}$ & $\begin{array}{l}<15 \mathrm{ml} / \mathrm{min} / 1,73 \mathrm{~m}^{2} \\
6-7 \mathrm{mg} / \mathrm{dL} \text { Creatinina } \\
10 \mathrm{ml} / \mathrm{min}\end{array}$ & $\begin{array}{l}\text { Siviero et al, } 2013^{14} \\
\text { Bastos et al } 2011^{6} \text {. } \\
\text { Barbosa et al, } 2012^{15} \\
\text { Bastos et al } 2011^{7} \text {. } \\
\text { Barbosa et al,2012 } \\
\text { Peixoto e Mamounier, } \\
2012^{16} \text {. }\end{array}$ \\
\hline Cistatina C & $70-90 \mathrm{ml} / \mathrm{min}$ & $\begin{array}{l}\text { Gabriel et al, } 2011^{21} \\
\text { Spanaus. Et al } 20111^{22}\end{array}$ \\
\hline Microalbuminúria & $300 \mathrm{mg} / 24$ horas & $\begin{array}{l}\text { Bastos et al 20117; } \\
\text { Siviero et al } 2013^{14} \\
\text { Barbosa et al } 2012^{15}\end{array}$ \\
\hline $\begin{array}{l}\text { Elementos anormais do } \\
\text { sedimento, urinálise } \\
\text { (EAS), Químico, Físico e } \\
\text { Microscópico }\end{array}$ & $\begin{array}{l}\text { Densidade = } 1010 \text { a } 1020 \text {, não existe } \\
\text { elementos anormais na densidade; } \\
\mathrm{PH}=(\text { de } 4,5 \text { a } 8,0) \text {; Proteína=expressa } \\
\text { em cruzes de } 1-4 \text {, Glicose; Nitrito, } \\
\text { Cetonas; Bilirrubina; } \\
\text { Urobilinogênio=são realizados com } \\
\text { tiras reagentes. }\end{array}$ & $\begin{array}{l}\text { Peixoto, } 2012^{16} \\
\text { Brasil, } 2013^{44}\end{array}$ \\
\hline $\begin{array}{l}\text { Exames de imagem e } \\
\text { biópsia renal }\end{array}$ & $\begin{array}{l}\text { Os ultrassons para observações de } \\
\text { fragilidades ósseas e a biópsia para } \\
\text { visualizar estruturas do órgão exemplo } \\
\text { hipertrofia dos néfrons. }\end{array}$ & $\begin{array}{l}\text { Peixoto e Lamounier } \\
2012^{16}\end{array}$ \\
\hline
\end{tabular}

à creatinina, pois podem detectar pequenas alterações na função renal, dentro da chamada zona cega da creatinina (falso biomarcador) 181920 .

A proteinúria é um termo genérico que engloba a excreção urinária de albumina e qualquer outro tipo de proteína. Já a palavra albuminúria refere-se única e exclusivamente à eliminação urinária de albumina, um marcador de lesão glomerular. Além disso, o termo microalbuminúria é utilizado quando a quantidade de albumina urinária está acima do normal, porém aquém dos níveis detectados nos testes de proteinúria total $^{21}$.

Atualmente 0 exame de elementos e sedimentos anormais da urina (EAS) encontra- se na rotina de todos os laboratórios por ser um exame barato e de fácil realização. $\mathrm{O}$ exame físico avalia a densidade, $\mathrm{pH}$, cor e aspecto da amostra onde fornecerá informações que auxiliará no resultado. $\mathrm{O}$ aspecto refere-se à transparência ou turvação, porém a turvação pode ser patológica quando possuem glóbulos vermelhos, glóbulos brancos e leveduras ou não patológica nos casos da presença de células epiteliais e muco. No caso da IRC a cor pode variar de incolor à avermelhada com presença de hematúria e a presença de elementos como os cilindros irão fornecer um aspecto turvo à urina. $\mathrm{Na}$ realização do exame químico utiliza-se a tira reativa com objetivo de analisar o $\mathrm{pH}$, proteínas, glicose, cetonas, 
bilirrubina, sangue, urobilinogênio, nitrito, densidade e leucócitos ${ }^{22}$.

$\mathrm{Na}$ insuficiência renal crônica (IRC) o paciente pode apresentar alguns distúrbios metabólicos alterando o pH da urina ácida, bem como níveis elevados de densidade, pois na lesão renal alguns elementos que retornam para a circulação e são detectados na urina devido ao trauma renal ${ }^{16}$

A biópsia renal é indicada em último caso para o diagnóstico auxiliar. Neste exame é possível observar as estruturas do órgão, principalmente a presença de hipertrofia dos néfrons, porém, trata-se de um procedimento invasivo que pode gerar riscos ao paciente devido a elevada vascularização dos rins. O diagnóstico de imagem também pode nortear a IRC, uma vez que é possível observar ossos fracos por causa da desmineralização devido à alteração do metabolismo do cálcio. ${ }^{16}$

Em relação aos tratamentos para a insuficiência renal crônica (IRC) variam de caso para caso sendo, na maior parte, apenas paliativos.
Todavia, contribuem para o alívio dos sintomas gerados pela doença renal. Atualmente, são três as modalidades de tratamento das quais dispõem os pacientes renais crônicos: a hemodiálise (HD), a diálise peritoneal (DP) e o transplante renal (TP) ${ }^{23}$.

$\mathrm{Na}$ tabela 2 apresentam-se os principais tratamentos substitutos para o paciente portador da insuficiência renal crônica, de acordo com as respectivas referências.

Antigamente, a hemodiálise (HD) tinha como objetivo apenas evitar a morte por hipervolemia ou hiperpotassemia. Nos dias atuais, além da reversão dos sintomas urêmicos, esse tratamento busca, a longo prazo, a redução das complicações, a diminuição do risco de mortalidade, a melhoria da qualidade de vida (QV) e a reintegração social do paciente, pois em pacientes com doença renal crônica, as falhas na adesão ou até mesmo a não adesão tem como consequência 0 aumento das complicações a da mortalidade (DRC) 2425 .

Tabela 2. Tratamentos não medicamentoso realizado para pacientes renais crônicos.

\begin{tabular}{|c|c|c|}
\hline Tratamento & $\begin{array}{r}\text { Processo } \\
\end{array}$ & Referência \\
\hline Hemodiálise & $\begin{array}{l}\text { Pacientes renais crônicos em tratamento } \\
\text { hemodialítico comumente necessitam de } \\
\text { pelo menos três sessões semanais de } \\
\text { terapia, com duração de aproximadamente } \\
\text { quatro horas cada. }\end{array}$ & $\begin{array}{l}\text { Silva, et al } 2011^{23} \\
\text { Frazão, et al , } 2011^{24}\end{array}$ \\
\hline Diálise peritonial & $\begin{array}{l}\text { A diálise peritonial (DP) é um processo no } \\
\text { qual um líquido especial, denominado } \\
\text { dialisato, é injetado na cavidade peritonial e } \\
\text { extrai no sangue do paciente, por difusão ou } \\
\text { por osmose, as substâncias tóxicas ou em } \\
\text { excesso que se encontrem no organismo. }\end{array}$ & $\begin{array}{l}\text { Barbosa et al } 2013^{13} \\
\text { Silveiro et al }{ }^{14}\end{array}$ \\
\hline Transplante renal & $\begin{array}{l}\text { O transplante renal é um procedimento } \\
\text { cirúrgico que consiste na transferência de } \\
\text { um rim saudável de uma pessoa para outra } \\
\text { com DRT, o objetivo é compensar ou } \\
\text { substituir a função que um órgão doente não } \\
\text { pode mais desempenhar. }\end{array}$ & $\begin{array}{l}\text { SNB, } 2013^{28} \\
\text { Garcia et al, } 2015^{45}\end{array}$ \\
\hline
\end{tabular}


A hemodiálise, é o tratamento dialítico mais utilizado na atualidade, é um processo que remove solutos acumulados em pacientes com perda total, ou quase total, da função renal. Apesar dos benefícios da HD, as condições impostas pela doença e pelo próprio tratamento dialítico resultam em uma série de alterações orgânicas, com complicações agudas, crônicas e nutricionais ${ }^{25}$.

A diálise peritoneal é uma opção de tratamento através do qual o processo ocorre dentro do corpo do paciente, a influência do procedimento dialítico sobre 0 metabolismo energético e proteico foi demonstrada pela redução de aminoácidos plasmáticos e síntese intracelular de proteína muscular, ocorrendo a proteólise muscular na tentativa de manter a concentração plasmática de aminoácidos. Esses eventos resultam em estado catabólico, que se estende até duas horas após a sessão de diálise. Essa cadeia é acompanhada por aumento no gasto de energia 2627 .

O transplante renal é reconhecido como um grande avanço na Medicina moderna, que fornece anos de vida com alta qualidade para pacientes com insuficiência renal irreversível. O transplante renal, quando aplicado adequadamente, é o tratamento de opção para pacientes com doença renal em estágio terminal (ESRD), devido aos baixos custos e aos melhores resultados. Países em desenvolvimento frequentemente têm taxas baixas de transplante, não só por esses diversos fatores de interação, mas também pela infraestrutura inferior e mão de obra treinada insuficiente ${ }^{2845}$.

Quando se trata da escolha do esquema terapêutico medicamentoso adequado, esse consiste em um processo individualizado, dessa forma, é possível promover a diminuição da morbidade e da mortalidade, entretanto, a não adesão ao tratamento farmacológico representa um importante obstáculo à obtenção dos resultados terapêuticos desejados, resultando em falha terapêutica e em sofrimento desnecessário, hospitalzações ou mesmo a morte precoce a adesão à terapêutica medicamentosa é um fenômeno sujeito à influência de múltiplos fatores que afetam diretamente o paciente ${ }^{23}$.

$\mathrm{Na}$ tabela 3 apresentam-se a farmacoterapêutica utilizada no cotidiano dos pacientes portadores da insuficiência renal crônica (IRC) de acordo com as referências analisadas.

O tratamento padrão para complicações da doença renal crônica como no caso da anemia, atualmente, consiste na administração de agentes estimulantes da eritropoiese e noripurum. Pacientes com insuficiência renal crônica (IRC) terminal em programa de diálise, o emprego de eritropoietina demonstrou bastante eficiência e segurança nessa situação. Como a resposta de eritropoietina é primariamente determinada pela disponibilidade de ferro, e esse mineral pode ser perdido durante 0 procedimento de diálise, o uso apropriado da suplementação de ferro é um ponto chave para o sucesso da terapia com eritropoietina. É o fármaco mais utilizado na terapêutica, inclusive no Brasilio 31.

O rim é o principal órgão envolvido na produção de formas bioativas de vitamina $D$ a partir de precursores inertes. Consequentemente, a doença renal crônica é um importante fator de risco para o desenvolvimento de deficiência de vitamina $D$. Níveis baixos de vitamina $D$ têm sido associados a uma taxa de mortalidade elevada na população geral e em pacientes mantidos em programa de hemodiálise (HD). O uso terapêutico da Vitamina D tem demonstrado redução da morbimortalidade cardiovascular e infecciosa, dado este de grande 
Tabela 3. Farmacoterapêutica utilizada por pacientes renais crônicos.

\begin{tabular}{|c|c|c|}
\hline Medicamentos & Indicação & Referência \\
\hline Eritropoetina & $\begin{array}{l}\text { Manter a hemoglobina do paciente entre } \\
11 \mathrm{~g} / \mathrm{dL} \text { e } 12 \mathrm{~g} / \mathrm{dL} \text {, e o hematócrito entre } 33 \% \\
\text { e } 36 \% \text {, estabelecidos por meio da Portaria } \\
\text { SAS/MS no. } 226 / 2010 \text { - Protocolo Clínico e } \\
\text { nas Diretrizes Terapêuticas para o } \\
\text { Tratamento da Anemia na Insuficiência } \\
\text { Renal Crônica. }\end{array}$ & $\begin{array}{l}\text { Gurgel, et al, } \\
2012^{31}\end{array}$ \\
\hline $\begin{array}{l}\text { Sevelâmer (Princípio ativo: } \\
\text { cloridrato de sevelâmer } 800 \mathrm{mg} \text { em } \\
\text { base anidra. } \\
\text { Excipientes: Dióxido de silício } \\
\text { coloidal e ácido esteárico. } \\
\text { Revestimento: Hipromelose e } \\
\text { monoglicerídeo diacetilado } \\
\text { Corante de impressão: Óxido de } \\
\text { ferro preto, propilenoglicol e álcool } \\
\text { isopropílico). }\end{array}$ & $\begin{array}{l}\text { Para o paciente da hiperfosfatemia e os } \\
\text { pacientes com DRC os estágios avançados } \\
\text { concentração aos quelatos de fósforo à } \\
\text { base de cálcio. }\end{array}$ & Brasil, $2013^{44}$ \\
\hline Vitamina D & $\begin{array}{l}\text { Carência de vitamina D; osteodistrofia renal; } \\
\text { hipoparatiroidismo; raquitismo, Prevenção e } \\
\text { tratamento do hiperparatiroidismo } \\
\text { associado à insuficiência renal crônica }\end{array}$ & $\begin{array}{l}\text { Alves et al,2013 } \\
39\end{array}$ \\
\hline Ácido Fólico & $\begin{array}{l}\text { Anemias megaloblásticas e resgate do } \\
\text { metrotexate em quimioterapia e antídoto } \\
\text { para doses tóxicas de piremetamina ou } \\
\text { trimetropim e anticonvulsivantes }\end{array}$ & $\begin{array}{l}\text { Pedroso } \\
\text { Oliveira, } 2012^{41}\end{array}$ \\
\hline Vitamina b12 & $\begin{array}{l}\text { Cofatores nas reações enzimáticas do } \\
\text { metabolismo da homocisteína. }\end{array}$ & $\begin{array}{l}\text { Gusmão et.al } \\
2013^{30}\end{array}$ \\
\hline Vitamina c & $\begin{array}{l}\text { Síntese de colágeno, que é uma estrutura } \\
\text { importante na formação de ossos, cartilagem, } \\
\text { músculos e vasos sanguíneos. A vitamina C } \\
\text { também atua no sistema imunológico e no } \\
\text { sistema nervoso central. }\end{array}$ & Pinheiro, $2015^{44}$ \\
\hline Andrógeno & $\begin{array}{l}\text { Antigonadotrófico por supressão da } \\
\text { secreção hipofisária de gonadotrofinas, } \\
\text { aumenta níveis de C4 no plasma; }\end{array}$ & Pedroso, $2012^{41}$ \\
\hline Estatina & $\begin{array}{l}\text { Inibem a redutase da hidroximetilglutaril- } \\
\text { coenzima } A(H M G C o A) \text {, enzima reguladora } \\
\text { da síntese do colesterol, levando à depleção } \\
\text { do colesterol hepático, as estatinas também } \\
\text { diminuem a secreção hepática de } \\
\text { lipoproteínas para a circulação. }\end{array}$ & $\begin{array}{l}\text { Vargas TC, et al } \\
2013^{33}\end{array}$ \\
\hline Sulfato Ferroso & $\begin{array}{l}\text { Tratamento da anemia por deficiência de } \\
\text { ferro que decorre de privação alimentar, } \\
\text { perdas crônicas ou interferência na } \\
\text { absorção de ferro. Prevenção e tratamento } \\
\text { da anemia da gravidez. }\end{array}$ & Pedroso, $2012^{41}$ \\
\hline
\end{tabular}

importância, visto que estas são as principais causas de morte em pacientes com DRC $\begin{array}{lllll}32 & 33 & 34\end{array}$. As vitaminas hidrossolúveis são perdidas durante a diálise, além de a ingestão alimentar usualmente estar deficiente. A suplementação é recomendada principalmente para o complexo $\mathrm{B}$ (ácido fólico e piridoxina) e vitamina $C$. Um fato relacionado à deficiência de vitaminas hidrossolúveis é a aterosclerose, causa comum de morte de pacientes em diálise ${ }^{35}$. 
A vitamina $C$ tem despertado grande interesse para a sociedade científica devido às funções fisiológicas atribuídas a essa substância consideradas como auxiliares na manutenção de uma pele saudável e na melhoria das características gerais da pele. Como a vitamina $\mathrm{C}$ é hidrossolúvel, o seu risco de toxicidade é grave por consumo excessivo, já que todo o excesso é facilmente excretado na urina, e a possui uma gama de funções no organismo, entre as mais importantes estão a formação do colágeno, que é uma substância necessária para ossos, ligamentos, tendões, músculos, pele e vasos sanguíneos, absorção de ferro pelo organismo, síntese de noradrenalina, que é um neurotransmissor, síntese de carnitina, substância que auxilia no funcionamento das mitocôndrias, metabolismo das prostaglandinas, o que Ihe confere algum efeito anti-inflamatório, ação antioxidante ${ }^{36} 37$.

As estatinas são a classe de fármacos que mais reduz as concentrações de colesterol, lipoproteína de baixa densidade (LDL). De maneira geral, a eficiência e a relativa segurança demonstradas pelas estatinas tornaram esses medicamentos amplamente utilizados em todo o mundo, sendo atualmente um dos mais comercializados. Os principais lipídios no plasma humano são o colesterol, ésteres de colesterol, triglicerídeos, fosfolipídios e os ácidos graxos não esterificados. Apesar dos grandes avanços tecnológicos das últimas décadas no tratamento da doença renal crônica (DRC), a doença cardiovascular continua sendo a principal causa de morbidade e mortalidade nos pacientes renais crônicos, sobretudo na população em diálise 38394041 .

A deficiência de ferro ocorre quando a quantidade absorvida não é capaz de suprir a necessidade do organismo e/ou de repor a perda sanguínea adicional, mais em decorrência desse problema que atinge todos os portadores dessa patologia, encontra-se disponível o sulfato ferroso que é a forma de primeira escolha para administração de ferro, $20 \%$ é ferro elementar. A melhor absorção de outros sais não justifica a troca, pois nas doses preconizada o sulfato ferroso é igualmente eficaz e bem mais barato. A tolerância em crianças é boa com todos os sais. Existem casos de anemia ferropriva resistente ao tratamento com outros sais de ferro e que respondem a doses terapêuticas de sulfato ferroso 42 43.

\section{Conclusão}

A partir do levantamento de artigos científicos para esta pesquisa, observa-se que para o diagnóstico laboratorial da insuficiência renal crônica tem como marcador bioquímico principal a taxa de filtração glomerular (TFG), assim como a hemodiálise (HD) é o principal tratamento não medicamentoso para os portadores da doença renal crônica de acordo com os autores citados na pesquisa e o tratamento medicamentoso mais utilizado é a eritropoietina, pois os rins não produz na quantidade adequada esse importante hormônio, que é responsável por manter os níveis de hemoglobina no sangue em quantidades adequadas, tendo como consequência a anemia, foi encontrado também a estatina, o andrógeno e a vitamina B12, que juntos ajudam no metabolismo das reações enzimáticas, na diminuição do colesterol e no sistema nervoso central SNC).

Portanto, essas informações podem de forma direta contribuir tanto para os profissionais da saúde como para os farmacêuticos, podendo de alguma forma ajudar na terapia desses pacientes, sabendo como traçar medidas mais apropriadas e realizar um bom acompanhamento. 


\section{Referências}

1. Dalapicola MM. Incidência do diabetes Mellitus em Pacientes com Doença Renal Crônica em Hemodiálise. Revista Saúde e Desenvolvimento 2013; jul/dez; 4(2).

2. Lages JS. Controle e avaliação da doença renal crônica no sistema único de saúde, 2015.

3. Coutinho NPS. Avaliação da atenção básica ao paciente renal crônico no estado do Maranhão. Brasília. Tese [Doutor em ciências da saúde]. Universidade de Brasília. 2011

4. Oliveira CS, Silva EC, Ferreira LW, Skalinski LM. Perfil dos pacientes renais crônicos em tratamento hemodialítico. Revista Baiana de Enfermagem, Salvador, 2015, jan./mar 29(1): $42-49$

5. Castro EK, Gross CQ. Percepção Sobre a Doença Renal Crônica de Pacientes em Hemodiálise: Revisão Sistemática. Salud \& Sociedad 2013; ENERO - ABRIL; 4(1).

6. Bastos MG. DRC: Diagnóstico precoce, encaminhamento imediato e abordagem interdisciplinar estruturada para 0e3h6ra do desfecho em pacientes ainda não submetidos à diálise 2011; 33(1): 93-108.

7. Bastos MG. et al. Doença Renal Crônica no Paciente Idoso. Rev. HCPA 2011; 31(1): 5265.

8. Terra FS. et al. O portador de insuficiência renal crônica e sua dependência ao tratamento hemodialítico: compreensão fenomenológica. Rev. Bras. Clin. Med. 2010; 8(4): 306-10; ; 8(4): 306-10.

9. Lopes, JM. et al. Qualidade de vida relacionada à saúde de pacientes renais crônicos em diálise. Acta Paul Enferm. 2014; 27(3): 230-6.

10.Terra FS. et. al. As principais complicações apresentadas pelos pacientes renais crônicos durante sessões de hemodiálise. Rev. Bras. Clin. Med. 2010, 8(3): 187-92.

11.Zambra B, Huth A. Terapia Nutricional em Pacientes Portadores de Insuficiência Renal Crônica em Hemodiálise. Revista Contexto \& Saúde ljuí editora Unijuí, 2010; jul./dez.; 10(19): 67-72.

12. Bastos MG, Kirsztajn GM. Doença renal crônica: importância do diagnóstico precoce, encaminhamento imediato e abordagem interdisciplinar estruturada para melhora do desfecho em pacientes ainda não submetidos à diálise. $\mathrm{J}$ Bras. Nefrol. 2011; 33(1): 93-108.

13.Barbosa AGC. et. al. O Significado da Doença Renal Crônica para o Paciente em Tratamento Hemodialítico: A Qualidade e Expectativa de Vida nos Aspectos da Humanização em Enfermagem. Revista Científica do Unisalesiano - Lins - SP, 2010; jul/dez 1(2).

14. Silveiro P, Machado CJ, Rodrigues RN. Doença renal crônica: Um agravo de proporções crescentes na população brasileira. Belo Horizonte. [Texto para discussão]. Universidade Federal de Minas Gerais, 2013.

15.Barbosa KRA. et. al. Indicação de Hemodiálise de Emergência em uma Unidade de Terapia Intensiva de um Hospital da Cidade de Atibaia-SP. Relato de Pesquisa. Sciense in Health. 2012; set-dez; 3(3): 131-8.

16. Peixoto EF, Lamounier TAC. Métodos laboratoriais para a identificação da insuficiência renal crônica. Acta de Ciências e Saúde 2012; 2(1).

17. Medeiros AJS, Medeiros EMD. Desafios do Tratamento Hemodiálitico para o Portador de Insuficiência Renal Crônica e a Contribuição da Enfermagem. Revista Brasileira de Educação e Saúde. 2013; janmar; 3(1): 1-10.

18.Costa LG. Caracterização do estado nutricional de portadores de insuficiência renal crônica em tratamento hemodialítico no distrito federal. Ceilância. Dissertação. [Mestre em nutrição clínica]. Faculdade de Brasília. 2015.

19.Sgnaolin V, Prado AE, Figueiredo L. Adesão farmacológica de pacientes em hemodiálise, J Bras Nefrol 2012; 34(2):109-116.

20. Ribeiro PRS, Batista TS. Adesão ao Tratamento Farmacológico AntiHipertensivos de Pacientes em Hemodiálise. Ver Ciênc. Básica Apl., 2015; 36(2):201-212.

21.Gabriel IC, Nishida SK, Kirsztajn GM. Cistatina $C$ sérica: uma alternativa prática para avaliação de função renal? J. bras. Nefrol. 2011, 33(2): 261-267.

22.Spanaus KS. et al. Creatinina sérica, cistatina $C$ e proteína $\beta$-traço no estadiamento diagnóstico e na predição da progressão da doença renal crônica não diabética. J. Bras. Patol. Med. Lab. 2011; $47(1)$. 
23. Silva GE. et al. Qualidade de Vida do Paciente Renal Crônico em Tratamento Hemodialítico em Dourados - MS. Psicol. inf. 2011; dez, 15(15).

24.Frazao CMFQ. et al. Qualidade de paciente Submetido à Hemodiálise. Rev. enferm. UERJ, Rio de Janeiro, 2011 out/dez; 19(4): 577-82.

25. Grincenkov FRDS, Fernandes N, Chaoubah A, Bastos K, Qureshi AR, Pécoits-Filho R, Bastos MG. Fatores associados à qualidade de vida de pacientes incidentes em diálise peritoneal no Brasil (BRAZPD). 2011, J. bras. nefrol, 33(1), 38-44.

26. Rocha PN, Sallenave M, Casqueiro V, Campelo Neto B, Presídio S. Motivo de escolha de diálise peritoneal: exaustão de acesso vascular para hemodiálise?. J. bras. Nefrol. 2010, 32(1): 23-28.

27. Abrahão SS, Ricas J, Andrade DF, Pompeu FC, Chamahum L, Araújo TM, Lima EM. Estudo descritivo sobre a prática da diálise peritoneal em domicílio. J. bras. Nefrol. 2010, 32(1): 45-50.

28.SBN. Protocolo clínico e diretrizes terapêuticas para 0 tratamento de hiperparatireodismo secundário em pacientes em doença renal crônica. 2013, 139.

29.Guimarães LRM, Ferreira AA. Caracterização e tratamento de anemia em pacientes com deficiência com insuficiência renal crônica. V Mostra Interna de Trabalhos de Iniciação Científica, 2010, Out.

30. Sociedade Brasileira de Nutrição Parenteral e Enteral. Terapia Nutricional para Pacientes em Hemodiálise Crônica. 2011, Ago.

31.Gurgel TC. et al. Utilização de eritropoetina por pacientes incidentes em hemodiálise no Sistema Único de Saúde Brasil, 2002-2003. Cad. Saúde Pública, 2012; mai., 28(5): 856868.

32. Guedes VPO. Farmacologia clínica dos antidislipidémicos. Portugal. Monografia [Mestrado integrado em Medicina]. Universidade do Porto. 2012.

33. Vargas TC, Limberger JB. Tratamento Famacológico com Estatinas: Uma Revisão Sistemática. Disciplinarum Scientia. Série:
Ciências da Saúde, Santa Maria, 2013; 14(2): 175-187.

34.Barancelli G, et al. Associação entre marcadores inflamatórios e lipídicos como preditores de risco cardiovascular em pacientes com insuficiência renal crônica que realizam hemodiálise. CEP 99970: 000.

35.Bonfim MR, et al. Caracterização do Tratamento Medicamentoso com Estatinas em Unidade Básica de Saúde. Medicina (Ribeirão Preto) 2013; 46(1): 47-55.

36. Filho AJI, Melamed ML. Vitamina D e doença renal. $O$ que nós sabemos e o que nós não sabemos. J Bras Nefrol 2013; 35(4):323-331.

37. Diniz HF. et al. Insuficiência e deficiência de vitamina $D$ em pacientes portadores de doença renal crônica. J Bras Nefrol 2012; 34(1):58-63.

38. Ellerkmann $C D$. et al. Perfil da vitamina $D$ em pacientes renais crônicos em tratamento hemodialítico em Mogi das Cruzes. Artigo [Internet] 2012. [Acessado em 15 de jun 2016].

39.Alves M. et. Al. Importância na Avaliação Laboratorial Rev. Port. Endocrinol. Diabetes Metab. 2013; 8(1):32-39.

40.Cançado RD, Chiattone CS. Anemia ferropênica no adulto-causas, diagnóstico e tratamento. Rev Bras Hematol Hemoter. 2010, 32(3), 240-6.

41.Pedroso ERP, Oliveira RG. Blackbook Clínica Médica. 1ํed. Belo Horizonte. Editora Ltda. 2012.

42. Anvisa. Fundação para o remédio popular: Furnp. Bula para o paciente. [internet] 2012. [Acessado 14 de jun 2016] Disponível em: www.anvisa.gov.br/datavisa/fila_bula/frmVi sualizarBula.asp?pNuTransacao.

43. Caye MT. et. al. Utilização da vitamina $C$ nas alterações estéticas do envelhecimento cutâneo. Artigo [internet] 2010.

44.Brasil. Ministério da Saúde. Nota técnica №332/2013. Brasília. 2013.

45.Garcia GG, et al. O papel global do transplante renal. J Bras. Nefrol. 2012; 34(1): 1-7. 